\title{
AUGMENTATION QUOTIENTS OF INTEGRAL GROUP RINGS
}

\author{
JOONGUL LEE
}

ABSTRACT, The structure of $I_{G}^{n} / I_{G}^{n+1}$ for finite abelian group $G$ is investigated.

\section{Two questions}

Let $G$ be a finite abelian group, $\mathbb{Z}[G]$ the integral group ring of $G$ and $I_{G}$ the augmentation ideal of $\mathbb{Z}[G]$, i.e., the kernel of the map from $\mathbb{Z}[G]$ to $\mathbb{Z}$ sending each group element to 1 . We define $Q_{n}(G)=I_{G}^{n} / I_{G}^{n+1}$ for $n \geq 1$ and set $Q_{0}(G)=\mathbb{Z}$.

It has been proved in [1] that there exist an integer $n_{0}$ such that $Q_{n}(G) \cong$ $Q_{n+1}(G)$ for all $n \geq n_{0}$. The eventually stabilized group $Q_{n_{0}}(G)$ is called the augmentation terminal of $G$ and is denoted by $Q_{\infty}(G)$.

Define $Q_{G}=\lim H$ where direct limit is taken over all cyclic subgroups $H$ of $G$ with respect to inclusion maps. Hales has proved in [3] that $Q_{\infty}(G) \cong Q_{G}$ for all finite abelian group $G$. His proof is of computational nature, and no isomorphism between $Q_{\infty}(G)$ and $Q_{G}$ is given. Hales also determined the structure of the associated graded ring

$$
\operatorname{gr}(\mathbb{Z}[G])=\bigoplus_{n=0}^{\infty} Q_{n}(G) .
$$

One may attempt to construct a natural isomorphism between $Q_{\infty}(G)$ and $Q_{G}$ in the following way. Using duality of finite abelian groups, one may view $Q_{G}=\lim G / H$ where (and from now on) inverse limit is taken over all cyclic quotient groups $G / H$ of $G$ with respect to natural projections. It is simple to see that $Q_{n}(G)$ is cyclic with order $|G|$ when $G$ is cyclic, hence $Q_{G} \cong \lim Q_{n}(G / H)$ for all $n$. Now let $\phi_{n}: Q_{n}(G) \rightarrow \lim Q_{n}(G / H)$ which is induced from natural map.

One may ask

Received June 1, 2007.

2000 Mathematics Subject Classification. 16S34.

Key words and phrases. group ring, augmentation ideal.

This work was supported by 2005 Hongik University Research Fund. 
Question 1. Is $\phi_{n}$ bijective for $n \geq n_{0}$ ?

A related question, raised by C. Popescu and D. Burns, is

Question 2. Is $\phi_{n}$ injective for all $n$ ?

The affirmative answer to Question 2 would give us a simple proof of a conjecture of Gross (cf. [2]) on special values of Abelian $L$-functions. The conjecture predicts a congruence relation which relates the Stickelberger element in $\mathbb{Z}[G]$ with the class number and the generalized regulator in $\mathbb{Z}[G]$ modulo $I_{G}^{n}$ for some suitable $n$. Both sides of the conjecture behaves functorially with respect to natural projection from $G$ to $G / H$, and the conjecture is fully proved for cyclic extensions. Therefore, one can say that the conjecture is true up to the kernel of $\phi_{n}$. In fact, the conjecture is fully proved for function fields, and for number field case it is enough to prove the conjecture when $G$ is a 2-group. Therefore we focus on the 2-groups in this paper.

Both questions have affirmative answers when $G$ is cyclic (for trivial reason) and more interestingly when $G$ is elementary (i.e., of prime exponent), as shown by Passi in [5]. Unfortunately, in general both have negative answers - Hayward was the first (cf. [4]) to observe this fact, using a computer program to compute the kernel of $\phi_{n}$. His computation suggests that the size of kernel of $\phi_{n}$ is quite small in general. The author finds it odd that the natural map between two isomorphic groups is not an isomorphism.

\section{An example}

Here we discuss an example which shows that $\phi_{n}$ is not injective in general.

Let $G=\mathbb{Z} / 16 \mathbb{Z} \times \mathbb{Z} / 16 \mathbb{Z} \cong\langle\sigma\rangle \times\langle\tau\rangle$ and let $\alpha=\sigma-1, \beta=\tau-1$ in $\mathbb{Z}[G]$. Let $\eta=\alpha^{8} \beta^{4}-\alpha^{4} \beta^{8}=\alpha^{4} \beta^{4}\left(\alpha^{4}-\beta^{4}\right)$. We will show that $\bar{\eta} \neq 0 \in Q_{12}(G)$ and that $\phi_{12}(\bar{\eta})=0$.

Hales showed (cf. [3], Corollary 2) that $\operatorname{gr}(\mathbb{Z}[G]) \cong \mathbb{Z}[\alpha, \beta] / J$, where $J$ is generated by $16 \alpha, 16 \beta$ and $2^{k}\left(\alpha^{2^{4-k}} \beta^{2^{3-k}}-\alpha^{2^{3-k}} \beta^{2^{4-k}}\right)$ for $k=0,1,2,3$. Considering modulo 2 , we conclude that $\eta \notin J$ hence $\bar{\eta} \neq 0 \in Q_{12}(G)$.

In order to show $\phi_{12}(\bar{\eta})=0$, one needs to show that for every projection $\psi: G \rightarrow \mathbb{Z} / 16 \mathbb{Z}$, the induced map $\bar{\psi}: \operatorname{gr}(\mathbb{Z}[G]) \rightarrow \operatorname{gr}(\mathbb{Z} / 16 \mathbb{Z})$ kills $\bar{\eta}$. Choose a generator $\pi$ of $\mathbb{Z} / 16 \mathbb{Z}$, and set $\gamma=\pi-1 \in \mathbb{Z}[G]$. It is easy to see that $\operatorname{gr}(\mathbb{Z} / 16 \mathbb{Z}) \cong \mathbb{Z}[\gamma] /(16 \gamma)$. If $\psi$ sends $\sigma$ to $\pi^{a}$ and $\tau$ to $\pi^{b}$, the induced map $\bar{\psi}$ sends $\alpha$ to $a \gamma$ and $\beta$ to $b \gamma$. Therefore, it eventually boils down to showing that $a^{4} b^{4}\left(a^{4}-b^{4}\right) \equiv 0(\bmod 16)$ for all $a, b \in \mathbb{Z}$. The congruence relation clearly holds if either $a$ or $b$ is even, and when both $a$ and $b$ are odd, $a^{4} \equiv b^{4} \equiv 1$ ( $\bmod 16)$, hence the relation also holds.

\section{A result}

Let $G=\mathbb{Z} / 4 \mathbb{Z} \times \mathbb{Z} / 4 \mathbb{Z}$. We show that $\phi_{n}$ is injective for all $n$.

By abuse of notation we will adopt the same notations as in previous section, i.e., $G \cong\langle\sigma\rangle \times\langle\tau\rangle, \alpha=\sigma-1, \beta=\tau-1$ etc. In this case, the result of 
Hales states that $\operatorname{gr}(\mathbb{Z}[G]) \cong \mathbb{Z}[\alpha, \beta] / J$ where $J$ is generated by $\left(\alpha^{4} \beta^{2}-\alpha^{2} \beta^{4}\right)$, $2\left(\alpha^{2} \beta-\alpha \beta^{2}\right), 4 \alpha$ and $4 \beta$.

Using the same method as in previous section, it is sufficient to show the following

Claim. Suppose $f(\alpha, \beta)$ is a homogeneous polynomial of degree $n$ with integer coefficients. If $f(a, b) \equiv 0(\bmod 4)$ for all $a, b \in \mathbb{Z}$, then $f(\alpha, \beta) \in J$.

Assume $n \geq 6$. As our goal is to show that $f \equiv 0(\bmod J)$, we have the freedom to modify $f$ by elements in $J$. Using the relation $\alpha^{4} \beta^{2} \equiv \alpha^{2} \beta^{4}$ $(\bmod J)$, we may assume that $f(\alpha, \beta)=c_{0} \beta^{n}+c_{1} \alpha \beta^{n-1}+c_{2} \alpha^{2} \beta^{n-2}+c_{3} \alpha^{3} \beta^{n-3}$ for some $c_{i} \in \mathbb{Z}, i=0,1,2,3$.

Letting $(a, b)=(0,1),(2,1),(1,1),(-1,1)$ respectively, we get $c_{0} \equiv 0 \quad(\bmod 4), c_{1} \equiv 0 \quad(\bmod 2), c_{1}+c_{2}+c_{3} \equiv c_{1}-c_{2}+c_{3} \equiv 0 \quad(\bmod 4)$. From these equations, we conclude that both $c_{2}$ and $c_{3}$ are even. Therefore, using the relation $2 \alpha^{2} \beta \equiv 2 \alpha \beta^{2}(\bmod J)$ we may assume that $f(\alpha, \beta)=$ $c_{1} \alpha \beta^{n-1}$, from which the conclusion follows readily.

The essentially same method can be applied individually when $n<6$ to prove the claim in full generality.

Corollary 1. The conjecture of Gross is true for extensions with Galois group $\mathbb{Z} / 4 \mathbb{Z} \times \mathbb{Z} / 4 \mathbb{Z}$.

\section{References}

[1] F. Bachmann and L. Grünenfelder, The periodicity by the graded ring associated with an integral group ring, J. Pure Appl. Algebra 5 (1974), 253-264.

[2] B. Gross, On the values of abelian L-functions at $s=0$, J. Fac. Sci. Univ. Tokyo Sect. IA Math. 35 (1988), no. 1, 177-197.

[3] A. W. Hales, Stable augmentation quotients of abelian groups, Pacific J. Math. 118 (1985), no. 2, 401-410.

[4] A. Hayward, On the graded ring associated to integral group rings, preprint (2002).

[5] I. B. S. Passi, Polynomial maps on groups, J. Algebra 9 (1968), 121-151.

Department of Mathematics Education

HONGIK UNIVERSITY

SEOUl 121-791, KoreA

E-mail address: jglee@hongik.ac.kr 